\title{
Research On Local Mesh Refine Method Based On Absolute Nodal Coordinate Formulation
}

\author{
Ruifeng Liu ${ }^{a}$, Gang He ${ }^{b}$, Jiawei Xu, Kang Gao, Hong Zhu \\ College of Mechanical \& Electrical Engineering, Hohai University; 210004, China \\ aemail: 953098793@qq.com, bemail: ihegang@163.com
}

\begin{abstract}
Keywords: Absolute Nodal Coordinate Formulation; Local Mesh Refinement; Stress Concentration; Fully Parameterized ANCF Plate Element
\end{abstract}

\begin{abstract}
In order to reduce the computation time and increase the convergence rate of absolute nodal coordinate formulation (ANCF), this paper gives a new local mesh refine method that can densify the mesh locally according to the degree of stress concentration. The mesh is divided into two kinds after comparing the maximum of the stress at each nodes with the average stress of the total fine mesh. The density of the new mesh is decided by the degree of stress concentration. The example that a rectangle plate fixed at four corner nodes with concentrate force applied at the center of the plate is simulated by fully parameterized ANCF plate element is given to verify our method.
\end{abstract}

\section{Introduction}

ANCF method uses absolute positions and gradients as nodal coordinates, which leads to a constant mass matrix and zero centrifugal and Coriolis forces [1], and allows for the use of general constitutive models in beam, plate, and shell elements. ANCF has been studied widely in the problems of large-rotation, large-deformation and the dynamic coupling between the rigid-body motion and the elastic deformation [2]. However, ANCF has more degrees of freedom than traditional FEM with the same element number, so the dynamics simulation based on ANCF will need more computation time. What's more, in order to get a convergent solution [3], fine mesh is need in the simulation of ANCF, and it will also increase the workload of computation.

In order to reduce the workload of ANCF simulation's computation and improve the simulation's accuracy, this paper gives a local mesh refine method, which is realized by densifying the area of stress concentration after comparing the maximum of every nodes' stress with the average stress of the fine mesh. An example with ANCF fully parameterized plate element is given to demonstrate the effectiveness of our method.

\section{Absolute nodal coordinate formulation (ANCF)}

In ANCF, the displacement field can be described as $\mathbf{r}(\mathbf{x}, t)=\mathbf{S}(\mathbf{x}) \mathbf{e}(\mathrm{t})$, where $\mathbf{r}$ is the global position vector of arbitrary point in the element, $\mathbf{x}=\left[\begin{array}{llll}x_{1} & x_{2} & x_{3}\end{array}\right]$ is the local coordinates defined in the element coordinate system, $\mathbf{e}$ is vector of the element nodal coordinates that consists of global position vector and gradient vector [4]; As the plate element [5] shown in Fig.1, the coordinate vector of plate element's node is defined as:

$\mathbf{e}=\left[\begin{array}{llll}\mathbf{e}^{1} & \mathbf{e}^{2} & \mathbf{e}^{3} & \mathbf{e}^{4}\end{array}\right]^{T}$



$\mathbf{S}$ is the element shape function matrix and can be written as

$\mathbf{S}=\left[\begin{array}{lll}S_{1} \mathbf{I} & S_{2} \mathbf{I} & S_{3} \mathbf{I} \ldots S_{16} \mathbf{I}\end{array}\right]$

Where $S_{1}, S_{2}, \ldots, S_{16}$ can be found in the work of Langerholc $M$ [5].

The Lagrange strain tensor can be defined as $\boldsymbol{\varepsilon}=\left(\mathbf{J}^{T} \mathbf{J}-\mathbf{I}\right) / 2$ [6], the position vector gradient matrix $\mathbf{J}$ is written as follows:

$$
\mathbf{J}=\partial \mathbf{r} / \partial \mathbf{x}
$$


For the elastic material, the second Piola-Kirchhoff stress can be calculated by generalized Hooke law as follows [7]:

$\boldsymbol{\sigma}=\mathbf{E}: \boldsymbol{\varepsilon}$

Where $\boldsymbol{\sigma}$ and $\boldsymbol{\varepsilon}$ is the second-order stress tensor and strain tensor respectively, $\mathbf{E}$ is the four-order elastic coefficient.

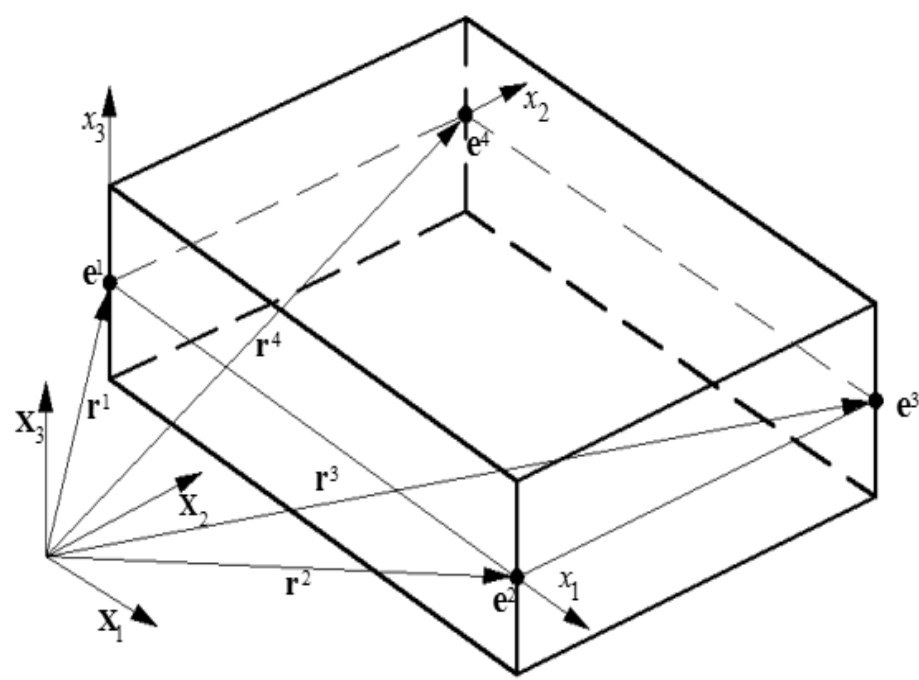

Fig.1. A fully parameterized ANCF plate element

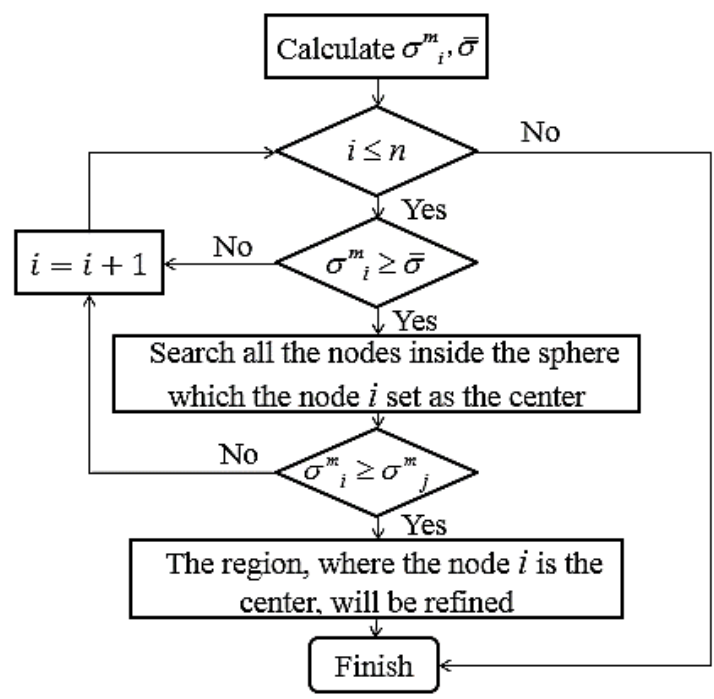

Fig.2. Refined region's searching process

\section{Local mesh refine method}

In this section the detailed steps of the local mesh refine method is introduced. Firstly, the characteristic of stress distribution is analyzed on a relatively fine mesh and stress concentration regions are searched in the mesh. A new mesh is generated with refinement in the concentration regions and smooth transition between different concentration regions. The degree of stress concentration directly determines density of nodes since there is a positive correlation between them [8].

The searching of refinement region.

The refinement process is mainly divided into three steps: (1) appropriately dividing the model into a uniform mesh; (2) calculating second Piola-Kirchhoff stress $\boldsymbol{\sigma}_{i}$ of each node in the mesh; (3) determining where the mesh should be densified according to the local stress distribution characteristic.

The maximum of absolute value of the component of node $i$ stress $\boldsymbol{\sigma}_{i}$ of is denoted as $\sigma_{i}^{m}$.

$\sigma_{i}^{m}=\max \left(\left|\boldsymbol{\sigma}_{i 1}^{m}\right|,\left|\boldsymbol{\sigma}_{i 2}^{m}\right|,\left|\boldsymbol{\sigma}_{i 3}^{m}\right|\right)$

Where $\boldsymbol{\sigma}_{i 1}^{m}, \boldsymbol{\sigma}_{i 2}^{m}, \boldsymbol{\sigma}_{i 3}^{m}$ represent three components of second Piola-Kirchhoff stress of node $i$.

$\bar{\sigma}$ represents the average value of $\sigma_{i}^{m}$ at all nodes, which can be calculated as

$$
\bar{\sigma}=\frac{1}{n} \sum_{i=0}^{n} \sigma_{i}^{m}
$$

Where $n$ represents the total number of nodes in the mesh.

As the Figure 2 shown, detailed steps of refined region's searching process:

Step 1: Calculating $\sigma_{i}^{m}$ and $\bar{\sigma}$ according to Equation (5) (6);

Step 2: Comparing $\sigma_{i}^{m}$ with $\bar{\sigma}$ in the given mesh direction; If $\sigma_{i}^{m} \leq \bar{\sigma}$, processing the next node in turn ; If $\sigma_{i}^{m}>\bar{\sigma}$, go to the Step 3; 
Step 3: The node which satisfies $\sigma_{i}^{m}>\bar{\sigma}$ is set as the center of sphere whose radius is $r_{0}$, and searching all the nodes inside the sphere;

Step 4: Comparing $\sigma_{j}^{m}$ of all nodes inside the sphere with $\sigma_{i}^{m}$ of center node, if center node $\sigma_{i}^{m}$ is greater than $\sigma_{j}^{m}$ of all other nodes; the region, where the node is the center, will be refined; otherwise processing the next node according to Step 2.

Where the size of $r_{0}$ and refined region is determined according to the value of $\sigma_{i}^{m} / \bar{\sigma}$, there is a positive correlation between them.

The distribution and connection of nodes.

The transition of mesh size of the refinement region and the non-refinement region should be smooth and uniform [9]. So the distribution of nodes between the refinement region and the non-refinement should have a smooth transition effect [10]. The method of arrangement is as follow:

(1)As the Figure 3 shown, nodes arrangement of plane model can be controlled by means of geometric progression; First of all, one should determine the length $l_{n}$ of the refined region element, the length $l_{0}$ of non-refinement region element and the length $L$ of transition region. The arrangement of nodes can be obtained by the following formula:

$$
\left\{\begin{array}{l}
l_{n}=l_{0} q^{n} \\
L=\left(l_{0}-l_{n} q\right) /(1-q)
\end{array}\right.
$$

Where $q$ is ratio coefficient of transition, $n$ is element number of transition region.

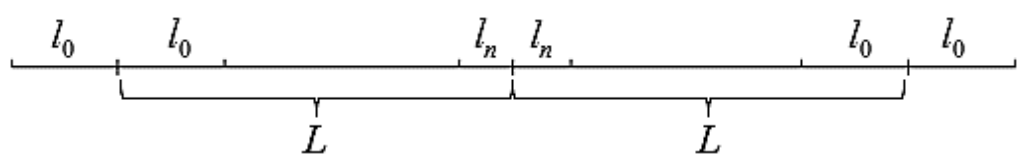

Fig.3. Transition in linear geometry

(2) As the Figure 4 shown, nodes arrangement of circular mesh can be controlled by the central angle. At the same time, the refined region and remained region should transit smoothly. The value of central angles can be expressed as follows:

$$
\left\{\begin{array}{l}
\alpha_{n}=\alpha_{0} q^{n} \\
\alpha=\left(\alpha_{0}-\alpha_{n} q\right) /(1-q)
\end{array}\right.
$$

Where $\alpha_{n}$ is central angle of the refined element, $\alpha_{0}$ is the central angle of the non-refined element, $\alpha$ is the central angle of the transition region, $q$ is ratio coefficient of transition $、 n$ is the element number in the transition region。

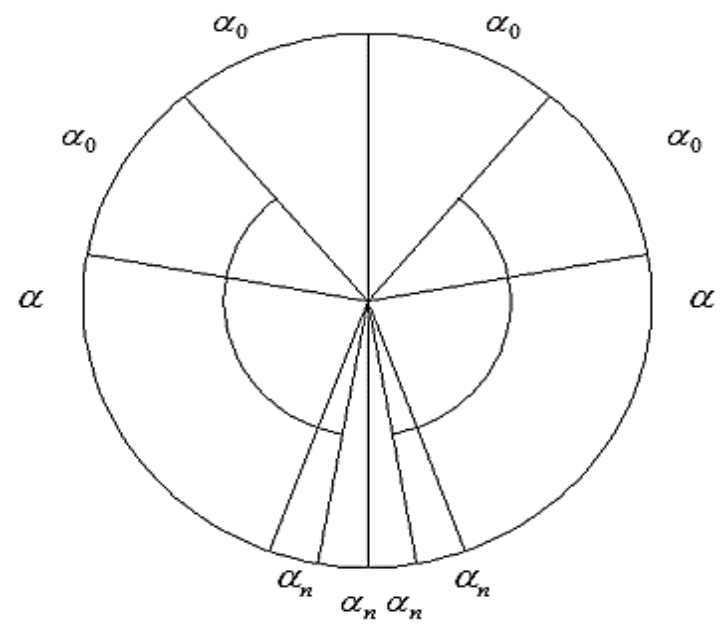

Fig.4. Transition in circular geometry 


\section{Example}

This section gives an example of a flat plate described by fully parameterized ANCF plate element to verify our method's effectiveness.

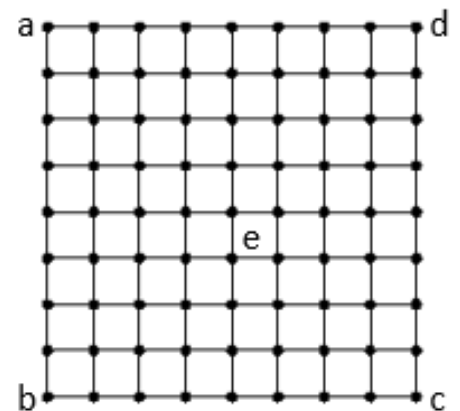

Fig.5. Initial mesh



Fig.6. The stress nephogram

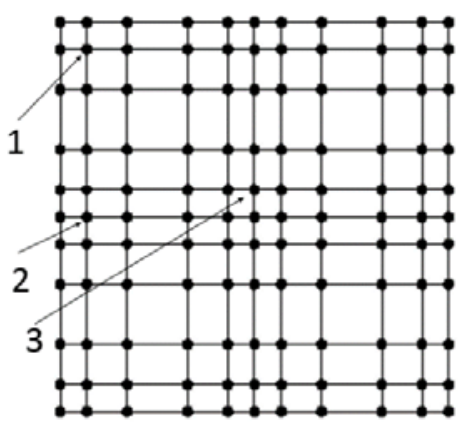

Fig.7. Mesh of local refinement

The initial mesh of the plate is shown in Figure 5. The size of the plate is $1 \mathrm{~m} \times 1 \mathrm{~m}$ and the thickness of the plate is $0.05 \mathrm{~m}$. The plate's density is $2000 \mathrm{~kg} / \mathrm{m} 3$, its Young's modulus is $0.2 \mathrm{MPa}$ and its Poisson ratio is set as 0 . The plate is fixed at four corner nodes labeled as a, b, c, d, and a concentrated force perpendicular to the plate is applied at the plate's center e. The plate is described by fully parameterized ANCF plate element. The simulation time is $0.5 \mathrm{~s}$.

The stress nephogram of the plate's neutral surface at $0.1 \mathrm{~s}$ is shown in Figure 6. After calculating the stresses of the mesh nodes, the stress concentration regions are located according to the local mesh refine method narrating in the third section, and the new mesh is generated as shown in Figure 7.

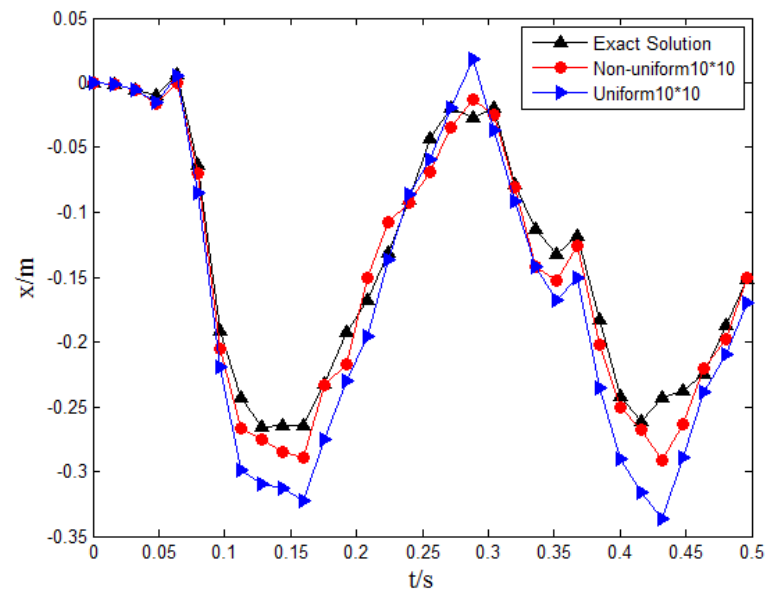

(a) Displacement of node 1

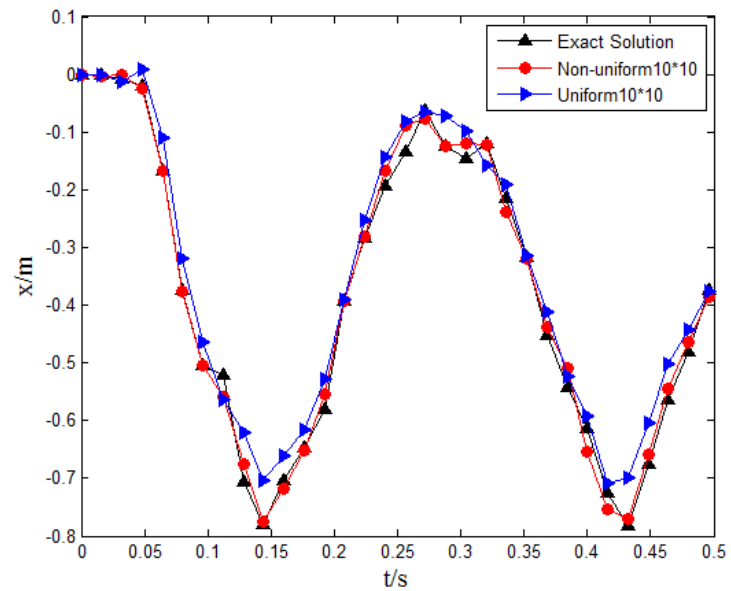

(b) Displacement of node 2

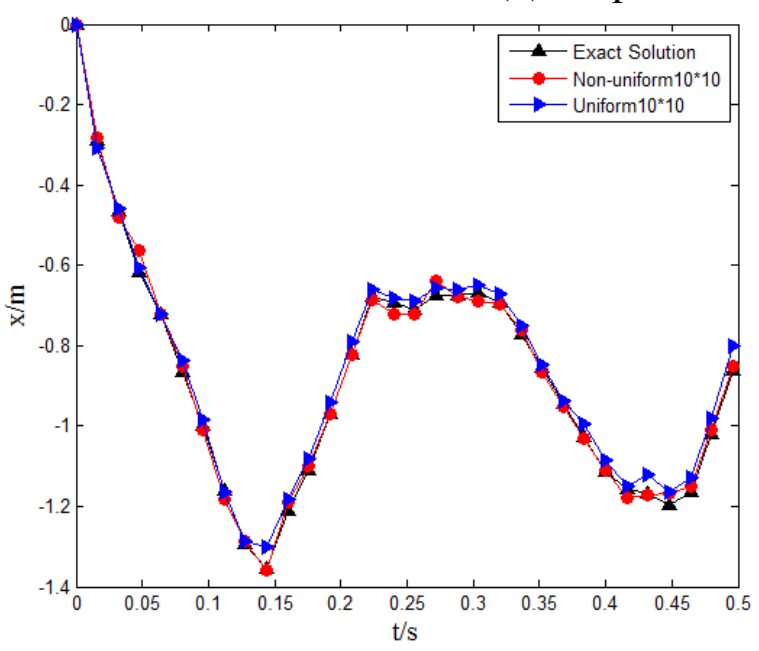

(c) Displacement of node 3

Fig .8. Displacement of node 
As Figure 7 shown, the displacements of 3 points from new Non-uniform mesh and the uniform mesh with the same numbers of nodes are calculated and compared with the results in the same points from the fine mesh which should have relatively more accurate result and are looked as an approximate exact solution [11]

Figure 8 shows the displacement time history curves of the three points in non-uniform distribution mesh, uniform distribution mesh and fine mesh. It can be seen clearly that the displacements of points in non-uniform mesh are more close to the fine mesh's corresponding displacements. The analysis results can prove that this method can improve the accuracy of ANCF dynamics simulation. When the same simulation accuracy can be achieved, the computational efficiency can be improved because of less elements and less degrees of freedom.

\section{Conclusion}

The paper proposes a local mesh refine method and is realized on fully parameterized ANCF plate element, which can densify the mesh locally according to the degree of stress concentration and this method has the advantage of reducing the simulation time and increasing the convergence rate of ANCF. The concentrate stress nodes are searching by comparing the stress with their neighbor nodes. The example of a rectangle plate fixed at four corner nodes with a concentrate force applied at the center is given to verify our method, and the simulation results show that this method can get higher accuracy compared with mesh of uniform mesh with the same number of elements. The next step of our research is to make this method more general that it can be used into more complex mesh.

\section{Acknowledgment}

This work was supported by National Natural Science Foundation of China (No. 51375141) and the Fundamental Research Funds for the Central Universities (No. 2013B18214). The authors would wish to thank the reviewers for their valuable comments.

\section{References}

[1] Shabana A A. Computational Continuum Mechanics [M]. Cambridge University Press, 2012.

[2] Shabana A A. Definition of the Slopes and the Finite Element Absolute Nodal Coordinate Formulation [J]. Multibody System Dynamics, 1997, 1(3):339-348.

[3] Matikainen M K, Valkeapää A I, Mikkola A M, et al. A study of moderately thick quadrilateral plate elements based on the absolute nodal coordinate formulation [J]. Multibody System Dynamics, 2013, 31(3):309-338.

[4] Shabana A A. ANCF Tire Assembly Model for Multibody System Applications [J]. Journal of Computational \& Nonlinear Dynamics, 2014, 10(2).

[5] Langerholc M, Slavič J, Boltežar M. A Thick Anisotropic Plate Element in the Framework of an Absolute Nodal Coordinate Formulation [J]. Nonlinear Dynamics, 2013, 73(1-2):183-198.

[6] Qiang T, Zhang Y, Chen L, et al. Advances in the absolute nodal coordinate method for the flexible multibody dynamics [J]. Advances in Mechanics, 2010, 40(2):189-202.

[7] Orzechowski G, Frączek J. Nearly incompressible nonlinear material models in the large deformation analysis of beams using ANCF [J]. Nonlinear Dynamics, 2015, 82(1):1-14.

[8] Wang Wei-xin, Deng Da-hua. An Adaptive Mesh Generation Algorithm Based on Geometric Features and Mechanical Properties [J]. Journal of Computer-Aided Design and Computer Graphics | J Comput-Aid Desig Comput Graph. 2001

[9] Yang X. High Quality Triangle Mesh Refinement Scheme in Adaptive Finite Element Method 
[J]. Journal of Mechanical Engineering, 2009, 45(8):292-297.

[10] Zhang W. Three Dimensional Entity Mesh Generation Algorithm [J]. Journal of Mechanical Engineering, 2009, 45(11):266-270.

[11] Xu S L, Cheng G D. Structural topology optimization based on adaptive mesh [J]. Journal of Dalian University of Technology, 2009, 49(4):469-475. 